\title{
Situation of the foot at risk due to leprosy in the municipality of Rio de Janeiro
}

\author{
Caroline Abreu ${ }^{\mathrm{a}}$, Marja Vilhena ${ }^{\mathrm{a}}$, Silvana Teixeira de Miranda ${ }^{\mathrm{a}}$, \\ Eduardo Alexander ${ }^{\mathrm{a}}$, Maria Cristina Dias da Silva ${ }^{\mathrm{a}}$, \\ Antônio José Ledo Alves da Cunha ${ }^{\mathrm{a}} \&$ Maria Katia Gomes ${ }^{\mathrm{a}}$ \\ ${ }^{a}$ Hospital Universitário Clementino Fraga Filho (HUCFF) - Universidade \\ Federal do Rio de Janeiro (UFRJ), Brazil; \\ ORCIDs: https://orcid.org/0000-0001-5107-602X; https://orcid.org/0000-0 \\ 001-6330-9740; https://orcid.org/0000-0003-0466-3322; https://orcid.org/0 \\ 000-0001-6638-0788; https://orcid.org/0000-0002-5345-0588; https://orcid \\ .org/0000-0003-3592-1849; https://orcid.org/0000-0002-1083-8531
}

Submitted 11 December 2020; Accepted 3 March 2021

\begin{abstract}
Summary Management of tibial neuritis is often hindered by silent neuropathy. The morbidity could be easily detected when the healthcare provider uses the systematic approach of simplified neurological evaluation recommended by the Brazilian Ministry of Health. Evolution from grade 1 disability (G1D) to grade 2 disability (G2D) is not uncommon after treatment with WHO/MDT owing to the development of plantar ulcers, osteomyelitis, and the need for surgical amputation.

Objective: We aimed to analyze the clinical course of patients with leprosy who presented with plantar anesthesia and G1D in the feet.

Methods: This is a retrospective longitudinal observational case study. We reviewed the records of Hansen's disease between 2004 and 2018 in four healthcare units in the Municipality of Rio de Janeiro. The sample comprised patients with leprosy (G1D or G2D) and anesthetic foot who underwent treatment and completed follow-up until discharge from WHO/MDT. Descriptive analysis and comparison between variables were performed using Fisher's exact test with $p<0.05$.

Results: Forty-nine patients were selected from 135 medical records. There was an increase in the physical disability grade in $20.4 \%$ of the cases due to the occurrence of plantar ulcers in $10.2 \%$ and in $49 \%$ of the patients with post-discharge complications. Chronic pain was the most reported symptom (22.4\%).

Conclusion: Inadequate and prolonged use of glucocorticoids for chronic pain, noncompliance in the use of orthosis and adequate footwear, and nonreferral for surgical decompression of the tibial nerve suggest the need for professional training in the therapeutic approach to nerve damage in leprosy.
\end{abstract}

Keywords: Leprosy, anesthetic foot, physical disability, chronic pain

Correspondence to: Caroline Abreu, Hospital Universitário Clementino Fraga Filho (HUCFF) - Universidade Federal do Rio de Janeiro (UFRJ), Brazil (e-mail: casandragabreu@gmail.com)

(C) The author(s). This article is Open Access under CC BY 4.0 


\section{Introduction}

Leprosy is a chronic infectious disease with an immunological background in which Mycobacterium leprae affects primarily the skin and peripheral nerves, with the development of sensory and motor changes and potential risk of permanent sequelae. Nerve damage is responsible for physical disabilities; therefore, systematic follow-up of patients and the search for effective therapies are necessary. 1,2

In Brazil, approximately $20 \%$ of all new cases present some type of disability at the time of detection; ${ }^{3}$ besides, there are cases of late diagnosis. It is noteworthy that $23 \%$ of patients exhibit disability grade progression even after discharge from WHO/MDT. ${ }^{4}$ In the last decade, studies have evaluated indirect clinical complications of leprosy, both related to physical disability and the psychosocial impact related to the stigma of the disease. ${ }^{5}$ Chronic pain in leprosy is one of the most frequent post-discharge complications (PDC); it is disabling and sometimes neglected, and its pathophysiology remains to be elucidated. ${ }^{6,7}$

Several studies have shown a higher frequency of foot disability than other disabilities due to leprosy ${ }^{8-10}$ such as plantar anesthesia, ulcerations, claw toes, and foot drop. Sensory alteration of the tibial nerve compromises the entire plantar region due to loss of protective sensation. Neuritis of the tibial nerve may be silent because of nerve dysfunction without manifestation of pain. ${ }^{11,12}$ Indeed, the absence of pain makes the diagnosis, treatment, and prevention of plantar ulcers challenging; hence, it is the most disabling of nonsymptomatic neuropathies. ${ }^{13}$

The recommended treatment by the Brazilian Ministry of Health and the World Health Organization (WHO) for leprosy-affected feet provided in primary healthcare units varies from self-care advice, such as the use of appropriate footwear, to occupational therapy (OT) with exercises and protective measures in daily activities. The provision of orthosis and insoles to improve body load distribution and thereby avoid areas of hyperpressure and ulcers, treatment of acute neuritis with corticotherapy, and adequate treatment of chronic pain are necessary. In cases of corticotherapy failure, in which no improvement of acute neuritis occurs after four weeks of treatment, the patient should be referred for surgical decompression of the tibial and/or fibular nerve. ${ }^{14}$

The aim of this study was to evaluate the healthcare measures carried out by the municipal health network of Rio de Janeiro regarding patients with leprosy classified as grade 1 of foot disability during and after MDT. By evaluating the progression of the at-risk foot in leprosy, we assessed the effectiveness or necessity of the systematic use of these actions to avoid further suffering for the patient. Thus, we expect to highlight the importance of monitoring the disabilities of individuals affected by leprosy, especially foot disabilities, either at the time of diagnosis, at discharge, or after discharge.

\section{General objective}

To analyze the clinical course of patients with leprosy having plantar anesthesia, grade 1 foot disability (G1D), diagnosed and followed in the healthcare units of the Municipality of Rio de Janeiro.

\section{Specific objectives}

- To identify the most frequent causes of an increase in disability grade among patients with leprosy at the time of discharge and in later. 
- To analyze the therapy provided by the basic healthcare network, focus on preventive measures, and its effectiveness in the treatment of the at-risk foot in terms of worsening disability and presence of post-discharge complications.

- To correlate the potential risk or protective factors for disability grade progression with post-discharge complications.

\section{Materials and methods}

This was a retrospective longitudinal observational case study using a quantitative approach that was conducted at four healthcare units, which adequately meets the objective of the study in question. ${ }^{3}$

The study field includes healthcare units located in the program areas of MRJ, namely, 3.1José Paranhos Fontenelle Polyclinic and Américo Veloso Municipal Health Center; 5.2Belizário Penna Municipal Health Center, and 5.3-Lincoln de Freitas Polyclinic. These units were composed of professionals specialized in the care and treatment of patients with leprosy, such as dermatologists, general practitioners, nurses, and occupational therapists.

The study sample consisted of patients with official mandatory notification records of Hansen's disease, classified as G1D or G2D, and were associated with anesthetic foot (grade 1 foot disability) who underwent treatment and complete follow-up until discharge from WHO/MDT in healthcare units of the city of Rio de Janeiro, from 2004 to 2018 (this study period followed the criteria of availability).

Patients after WHO/MDT treatment, classified as G1D or G2D at diagnosis, associated with grade 1 foot physical disability, i.e., anesthetic foot, and who were followed up in the municipal healthcare network were included.

Patients with diabetes mellitus and/or chronic alcoholism or a condition other than leprosy causing peripheral neuropathy; patients classified as G0D at the time of diagnosis; patients with insufficient data for medical record searching; patients transferred to other units, and patients who either died or abandoned the treatment were excluded.

After accessing data from the Municipal Health Department of Rio de Janeiro through notification records from the Information System for Notifiable Diseases (SINAN) of patients having leprosy G1D and G2D, we recruited four health units that had the highest number of notifications and provided the best facilities with a complete team for the care of the at-risk foot between January 2004 and January 2018. Subsequently, a comprehensive analysis of the medical charts of outpatient visits managed throughout the treatment by a multidisciplinary team of clinicians or dermatologists, nurses, physical therapists or occupational therapists was performed.

The following variables were selected and analyzed: sex; age at diagnosis; Madrid and Operational classifications of leprosy and disability grade at diagnosis and at discharge; leprosy reactions; initial clinical manifestation; treatment provided for the at-risk foot; progression of foot disability grade-worsening, improvement, or no change; causes of disability grade worsening and post-discharge complications.

The data collected were analyzed using the IBM SPSS statistics software, version 22 . Descriptive analysis of the data was performed using distribution of frequency, mean, and standard deviation. Fisher's exact test with $p<0.05$ was used to compare the variables.

This study complied with the ethical recommendations of resolution National Health Council (NHC) 466/12 and was submitted to and approved by the Research Ethics Committee (CEP) of the HUCFF-UFRJ under opinion no. 2.682.393 of May 29, 2018. 


\section{PD GRADE WORSENING AT} DISCHARGE

Dulcers

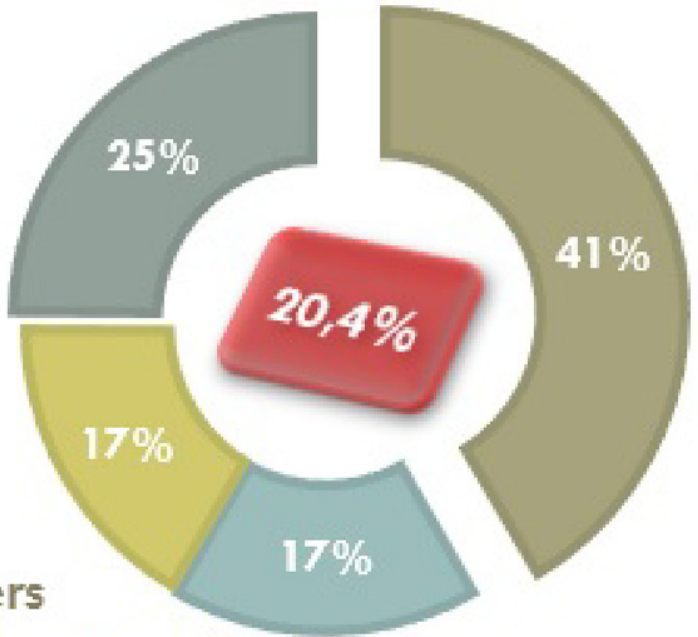

worsening of sensitivity

fallen foot

claw of fingers

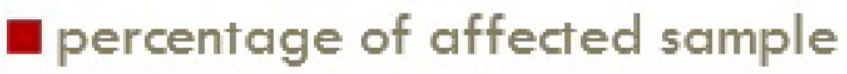

Figure 1. Total value and causes of disability grade worsening at discharge in the sample of patients with leprosy neuropathy in the lower limbs followed in secondary units of the city of Rio de Janeiro from 2004 to 2018. Source: own elaboration, 2020.

\section{Results}

A total of 135 medical records from the four selected primary healthcare units were evaluated, with 86 follow-up losses due to treatment abandonment (22 cases), transfer to another unit (31 cases), and insufficient data or presence of clinical exclusion criteria (33 cases); finally, 49 cases were analyzed. The characteristics of the study sample are shown below (Table 1). It is noteworthy that all patients had plantar anesthesia, classified as grade 1 foot disability, which was the population of interest.

All patients in the sample completed treatment with the WHO/MDT. The treatment provided for the at-risk foot as well as the foot disability grade at diagnosis and discharge were evaluated. The causes of foot disability grade worsening, which occurred in $20.4 \%$ of cases, and the complications after discharge, which occurred in $49 \%$ of cases (Figure 1/Figure 2), were also assessed.

The evaluation of the operational classification and disability grade at the time of diagnosis with episodes of leprosy reactions, worsening of disability grade and complications after discharge from MDT, as well as the correlation of treatment for the anesthetic foot with progression to worsening of disability grade and post-discharge complications were not statistically significant $(p>0.05)$. 
Table 1. Characterization of the sample of patients $(N=49)$ with leprosy neuropathy in the lower limbs followed in secondary units of the city of Rio de Janeiro (sex, duration of the disease, leprosy reactions, operational classification, disability grade at diagnosis, initial clinical presentation, and Madrid classification, from 2004 to 2018)

\begin{tabular}{|c|c|c|c|c|c|c|c|c|}
\hline Sex & $\mathrm{F}$ & M & Mean & DP & & & & \\
\hline$n(\%)$ & 30.6 & 69.4 & 1.69 & 0.46 & & & & \\
\hline Duration of the disease & $<6 \mathrm{~m}$ & $6-12 \mathrm{~m}$ & $>12 \mathrm{~m}$ & NA & Mean & $\mathrm{DP}$ & & \\
\hline$n(\%)$ & 14.2 & 10.2 & 38.7 & 36.7 & 25.2 & 29.9 & & \\
\hline Leprosy reactions & I & II & Mean & $\mathrm{DP}$ & & & & \\
\hline$n(\%)$ & 38.8 & 20.4 & 1.51 & 0.50 & & & & \\
\hline Operational classification & MB & PB & Mean & $\mathrm{DP}$ & & & & \\
\hline$n(\%)$ & 83.7 & 16.3 & 1.16 & 0.37 & & & & \\
\hline PD grade at diagnosis & 0 & 1 & 2 & Mean & DP & & & \\
\hline$n(\%)$ & 0 & 93.9 & 6.1 & 1.06 & 0.24 & & & \\
\hline Initial clinical presentation & Eye & Skin & Hand & Foot & & & & \\
\hline$n(\%)$ & 10.2 & 93.9 & 67.3 & 91.8 & & & & \\
\hline Mean & 1.10 & 1.94 & 1.67 & 1.92 & & & & \\
\hline DP & 0.30 & 0.24 & 0.47 & 0.27 & & & & \\
\hline Madrid classification & $\mathrm{D}$ & DT & DV & I & $\mathrm{T}$ & $\mathrm{V}$ & Mean & DP \\
\hline Percentage & 40.8 & 4.1 & 4.1 & 4.1 & 10.2 & 36.7 & 3.49 & 2.30 \\
\hline
\end{tabular}

$n(\%)$ : Percentage values. F: female; M: male; m: months; SD: standard deviation; NA: not assessed; MB: multibacillary; PB: paucibacillary; D: dimorphous; DT: dimorphous-tuberculoid; DV: dimorphous-virchowian; I: undetermined; T: tuberculoid; V: virchowian. Source: own elaboration, 2020.

Table 2. Correlations in the sample of patients with leprosy neuropathy in the lower limbs followed in secondary units of the city of Rio de Janeiro from 2004 to 2018. PD grade at diagnosis X plantar ulcer

\begin{tabular}{|c|c|c|c|c|c|c|}
\hline \multirow[t]{3}{*}{ PD grade at diagnosis } & \multicolumn{3}{|c|}{ PD grade worsening } & \multicolumn{3}{|c|}{ Complications after discharge } \\
\hline & \multicolumn{3}{|c|}{ Ulcer } & \multicolumn{3}{|c|}{ Ulcer } \\
\hline & No & Yes & $p$ value & No & Yes & $p$ value \\
\hline 1 & 93.5 & 6.5 & & 97.8 & 2.2 & \\
\hline 2 & 33.3 & 66.7 & 0.024 & 33.3 & 66.7 & 0.008 \\
\hline
\end{tabular}

Percentage values. PD grade: grade of physical disability. Source: own elaboration, 2020.

Table 3. Correlations in the sample of patients with leprosy neuropathy in the lower limbs followed in secondary units of the city of Rio de Janeiro from 2004 to 2018. Duration of disease X PDC

\begin{tabular}{cccc}
\hline Duration of disease & & PDC & $p$ value \\
\cline { 2 - 4 } & No & Yes & \multirow{2}{*}{0.032} \\
\hline$<6$ months & 57.1 & 42.9 & \\
6-12 months & 87.5 & 12.5 & 68.8 \\
\hline 12 months & 31.3 & 6 & \\
\hline
\end{tabular}

Percentage values. PDC: post-discharge complication. Source: own elaboration, 2020.

The analysis of the correlation between disability grade at diagnosis and ulcer development showed that among the patients with initial G2D, considering that they had grade 1 foot disability, $66.7 \%$ progressed to plantar ulcers until discharge $(p=0.024)$ and after discharge (0.008) (Table 2).

There was a statistically significant correlation between duration of disease and occurrence of post-discharge complications ( $p^{-}=0.032$ ). Thus, $68.8 \%$ of patients with manifestations of the disease for more than 12 months had complications after discharge from MDT (Table 3). 


\section{COMPLICATIONS AFTER DISCHARGE}

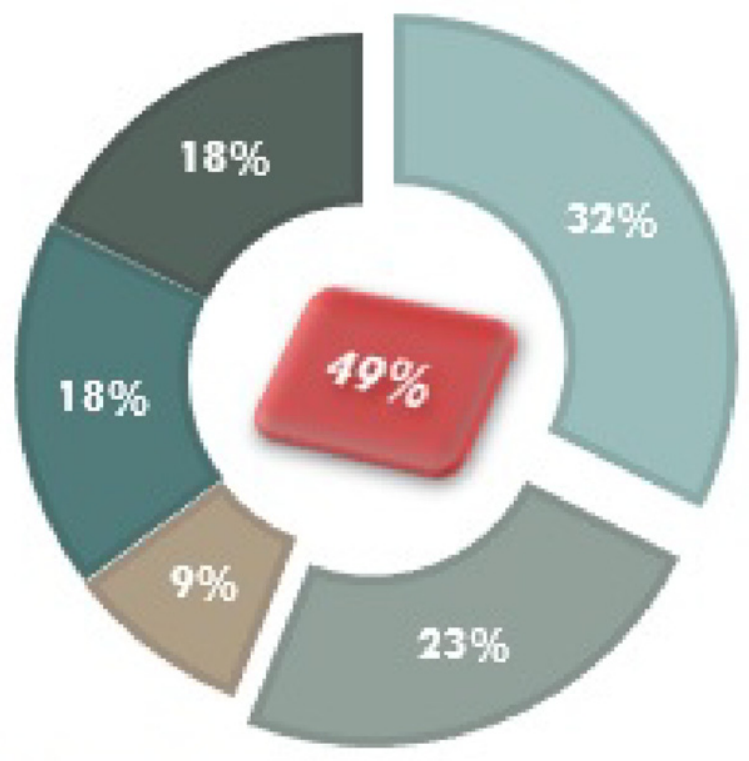

a chronic pain

口 chronic corticosteroid

alcers

neuritis

athers

- percentage of affected sample

Figure 2. Total value and causes of post-discharge complications in the sample of patients with leprosy neuropathy in the lower limbs followed in secondary units of the city of Rio de Janeiro from 2004 to 2018. Source: own elaboration, 2020.

The relationship between the treatment provided for the at-risk foot and possible causes of post-discharge complications were also investigated, including chronic pain complaints, the need for chronic corticosteroid use, neuritis, the occurrence of plantar ulcers, and others not related to leprosy. Statistically significant values were found in the preventive relationship between OT and chronic pain and chronic use of corticosteroids, i.e., among those who received OT, only $9.4 \%$ developed chronic pain $(p=0.005)$ and $6.2 \%$ required chronic corticosteroid therapy $(p=0.015)$. The other variables did not present a statistically significant correlation.

Lastly, the relationship between the possible causes of post-discharge complications was analyzed in an attempt to find a common cause or alteration between them. There was a statistically significant relationship between neuritis and chronic pain $(p=0.018)$ and between neuritis and chronic use of glucocorticoids (0.047) (Table 4). 
Table 4. Correlations in the sample of patients with leprosy neuropathy in the lower limbs followed in secondary units of the city of Rio de Janeiro from 2004 to 2018. Neuritis X chronic pain X chronic use GC X Ulcer X Others

\begin{tabular}{cccc}
\hline & \multicolumn{3}{c}{ Neuritis } \\
\cline { 2 - 4 } & No & Yes & $p$ value \\
\hline Chronic pain & $84 \%$ & $16 \%$ & 0.018 \\
No & $33.3 \%$ & $66.7 \%$ & \\
Yes & & & \\
Chronic use GC & $88.4 \%$ & $11.6 \%$ & 0.047 \\
No & $50 \%$ & $50 \%$ & \\
Yes & & & 0.67 \\
Ulcer & $93 \%$ & $7 \%$ & \\
No & $100 \%$ & $0 \%$ & 0.436 \\
Yes & & & \\
Others & $86 \%$ & $14 \%$ & \\
No & $100 \%$ & $0 \%$ & \\
Yes & & & \\
\hline
\end{tabular}

GC: glucocorticoids. Source: own elaboration, 2020.

\section{Discussion}

The sample of this study was similar to those described in the literature: mean age of 47.2 years, working individuals, and a predominance of men. ${ }^{6,14}$ With regard to disease duration, known to be related to poor prognosis, ${ }^{8,10} 38.7 \%$ experienced the disease for more than 12 months, which is comparable to the literature ${ }^{15}$ and confirms the failure of early detection of the disease in the municipality of Rio de Janeiro. Moreover, $66.8 \%$ of patients who had the disease for more than 12 months had some post-discharge complication from MDT, a statistically significant result that was not found in other studies. ${ }^{8}$

There was worsening of the disability grade in $20.4 \%$ of cases, a result similar to that found in the literature $(25 \%) .{ }^{8}$ Moreover, there was an improvement of $18 \%$ in this parameter at discharge, which is in agreement with studies that showed a reduction in the disability grade of $15 \% .^{4}$

The treatment provided to patients for the at-risk foot was in accordance with WHO recommendations, by regular monitoring during treatment with MDT and the foot sensory map was used to record the evolution of foot injury. ${ }^{16}$ Practices of self-care are taught under the guidance and supervision of the multidisciplinary team responsible for the treatment and follow-up of leprosy patients, mainly nurses and occupational therapists.

However, there was a low frequency of insoles and orthosis use for the treatment of anesthetic feet, which prevented adequate evaluation of the benefit on the study population. Only $6.1 \%$ of patients had access to this treatment because of the difficulty of referral due to the shortage of orthosis providers in the city of Rio de Janeiro. The use of insoles and orthosis has been recognized as contributing to a better load distribution on the anesthetic foot, preventing the occurrence of plantar ulcers. In some cases, this approach has been included in the conservative treatment of this condition. ${ }^{17}$

Additionally, glucocorticoids were prescribed only by health professionals in $83.7 \%$ of cases of foot disability, either for the treatment of reaction episodes, which occurred in $59.2 \%$ of patients, or for the treatment of neuritis. $41.5 \%$ of the patients who underwent chemical 
decompression had a good response to chemical decompression. We did not find a statistically significant relationship between the use of glucocorticoids and the assessed outcomes.

Many patients were maintained on glucocorticoids, which were probably initiated for the treatment of acute neuritis and subsequently inadequately maintained for the management of chronic pain. Absence of symptom resolution after four weeks of glucocorticoids therapy corresponds to treatment failure and is an indication for surgical treatment. This did not occur in this sample as no neurolysis was performed, given the absence of patient referral to surgery.

The listed variables related to plantar anesthesia are: (1) failure to identify neuritis, (2) failure of conservative treatment due to patients not wearing insoles or orthosis, (3) inability to identify chemical decompression failure with prolonged inadequate use, and (4) the lack of indication for tibial nerve neurolysis, associated with the statistically significant finding of plantar ulcers as a cause of disability grade worsening in $10.2 \%$ of cases. Accordingly, we may conclude that the prevention of this disability failed, mainly due to the absence of indication for neurolysis. Therefore, we agree with the authors ${ }^{18}$ who advocate decompression of the tibial nerve at the first signs of its involvement-local pain and loss of sensation-once silent neuropathy may be catastrophic, resulting in plantar ulcer, injury that is difficult to manage, and high morbidity.

Complications after discharge were assessed and deemed present in $49 \%$ of cases. They included chronic pain, chronic corticosteroid use, neuritis, plantar ulcer, and others not directly related to leprosy. They were more frequent than that reported in the literature, in which the rate of chronic complications from leprosy after discharge was $25.9 \%$. Chronic pain was the most important and most frequent finding in this sample, affecting $22.4 \%$ of patients. Its prevalence is not yet well documented, although some authors ${ }^{19-21}$ have reported a prevalence of $29 \%$ to $70 \%$. Data in the literature show that $75.4 \%$ of patients with chronic pain have been discharged ${ }^{19}$ and that tibial nerve injury was more common in patients with chronic pain $(90.4 \%)$ than in those who did not have pain $(68.2 \%){ }^{21}$

Among patients with neuritis as a post-discharge complication, $66.7 \%$ and $50 \%$ had chronic pain and were on chronic corticosteroid use, respectively, the results being statistically significant. Therefore, neuritis, caused by nerve damage, is the commonest cause of complications after discharge in leprosy. Another important statistically significant finding was that $75 \%$ of patients with chronic pain were also using corticosteroids chronically.

With regard to the treatment of the at-risk foot, OT was a preventive element both for chronic pain and for chronic corticosteroid use. This relationship was not observed or even assessed in the literature. ${ }^{21}$ Only $9.3 \%$ and $6.4 \%$ of patients who received OT had chronic pain and used corticosteroids chronically, with statistical significance. None of the other forms of treatment for the anesthetic foot evaluated in this study showed a significant statistical relationship, including corticosteroid therapy.

There was no correlation between worsening of disability grade and post-discharge complications, but there was a statistically significant correlation with plantar ulcers. The higher the initial grade of physical disability, the greater the chance of the patient presenting plantar ulcers, both as a cause of disability grade worsening at discharge and as a cause of complications after discharge. Importantly, $66.7 \%$ of patients classified as G2D progressed to plantar ulcers before and after discharge, and this worsening of grade was solely related to the foot.

In conclusion, the nonrecognition of failure of conservative treatment for neuritis perpetuated the use of chronic corticosteroids and the maintenance of neuritis and chronic pain after discharge; there was worsening of the disability grade due to the development of plantar ulcers 
related to patients not wearing orthosis and lack of indication for neurolysis, either due to lack of professional training or insufficiency of the regulatory system; OT was effective in preventing complications after discharge, especially chronic pain and, indirectly, neuritis and chronic use of glucocorticoids; the prevention of disabilities and complications after discharge from MDT are directly related to the therapeutic approach to nerve damage in leprosy.

\section{Review board approval}

Research Ethics Committee (CEP) of the HUCFF-UFRJ under Opinion No. 2.682.393 of May 29, 2018.

\section{Conflict of interest (COI)}

The authors report no conflict of interest.

\section{Funding}

No funding was provided for this research.

\section{Patient consent statement}

No patient consent was required.

\section{Acknowledgements}

None to declare.

\section{References}

1 Brasil; Ministério da Saúde; Secretaria de Vigilância à saúde. Manual de Prevenção e reabilitação em hanseníase. Brasília, DF, v. 1-4, n. 3a ed., rev. e amp, (2008).

2 Croft RP, Nicholls PG, Steyerberg EW et al. A clinical prediction rule for nerve-function impairment in leprosy patients. Lancet, 200(355): 1603-1606.

3 Opromolla DVA. Terapêutica de Hanseníase. Medicina, Ribeirão Preto, 1997; 30: 345-350.

4 Gonçalves SD et al. Fatores preditivos de incapacidades em pacientes com hanseníase. Rev Saude Publica, 2009; 43: 267-274.

5 World Health Organization. Global leprosy situation, 2010. In: Weekly Epidemiological Report, vol. 85, 2010; pp. 185-202. http://www.who.int/wer.

6 World Health Organization. Global leprosy update, 2014: Need for early case detection. In: Weekly Epidemiological Report, vol. 90, 2015; pp. 461-474. http://www.who.int/wer.

7 Walker SL, Lockwood DNJ. Leprosy. Clin Dermatol, 2007; 25: 165-172.

8 Monteiro LD et al. Incapacidades físicas em pessoas acometidas pela hanseníase no período pós-alta da poliquimioterapia em um município do Norte do Brasil. Cad saúde pública, rio janeiro, 2007; 23: 1187-1197.

9 Trindade MAB, Lima FD, Almeida RG. Incapacidades físicas em hanseníase no momento do diagnóstico: iavaliação das incapacidades. Hansen Int, 1987; 12: 21-28.

10 Finez MA, Salotti SR. Identification of the degree of impairment in leprosy patients through a simplified neurological evaluation. J Heal Sci Inst, 2011; 29: 171-175.

11 Job CK. Nerve damage in leprosy. Int J Lepr Other Mycobact Dis, 1989; 57: 532-539, 1989.

12 Richardus JH, Withington SG, Anderson AM et al. Treatment with corticosteroids of long-standing nerve function impairment in leprosy: a randomized controlled trial (TRIPOD 3). Lep Rev, 2003; 74: 311-318.

13 Garbino JA. Manejo clínico das diferentes formas de comprometimento da Neuropatia Hanseniana. In: Hansenologia internationalis, v. Special 98, n. Congress. 1996; pp. 93-99.

14 Helene LMF, Leão VM, Minakawa MM. The social situation and the presents of physical disabilites among leprosy patients registered at a public health center in São Paulo city. Hansen Int, 2001; 26: 5-13.

15 Ramos JMH, Souto FJD. Incapacidade pós-tratamento em pacientes hansenianos em várzea grande, estado de mato grosso. Rev Soc Bras Med Trop, 2010; 43: 293-297. 
16 Brasil; Ministério da Saúde; Secretaria de Vigilância em Saúde. Diretrizes para vigilância, atenção e eliminação da Hanseníase como problema de saúde pública (Manual técnico-operacional). Brasília, DF. p58 (2016).

17 Enna CD, Reed JK. The orthotic care of the denervated foot in hansen's disease. Orthosis Prothes, 1976; 30: 33-39.

18 Duerksen FA. Hanseníase e a neuropatia através da perspectiva de um cirurgião. Hansen, 2004; 29 : 41-45.

19 Raicher I, Stump PR, Baccarelli R et al. Neuropathic pain in leprosy. Clin Dermatol, 2016; 34: 59-65.

20 Reis FJ, Gomes MK, Saadi L et al. Chronic pain in leprosy: new aspects to be considered. Pain Manag, 2013; 3: $201-210$.

21 Ramos JM, Alonso-Castañeda B, Eshetu D et al. Prevalence and characteristics of neuropathic pain in leprosy patients treated years ago. Pathog Glob Health, 2014; 108: 186-190. 